\title{
Evaluation of postural stability in young and elderly women
}

\author{
Tomasz Marciniak, Ida Wiszomirska, Lidia IInicka \\ Department of Rehabilitation, Józef Piłsudski University of Physical Education, Warsaw, Poland
}

\begin{abstract}
Summary
Study aim: Assessment of postural stability performed on an unstable stabilometry platform. Comparison of the results obtained by two groups consisting of elderly (OW) (60+ years old) and younger women (YW).

Material and methods: Seventy-three female volunteers were divided into two groups: 40 young women (20.2 \pm 1.75$)$, and 32 elderly women $(68.3 \pm 7.43)$. Participants performed five stability tests on Biodex Balance System SD: three 20-second tries, the Postural Stability Test (PST) and the Fall Risk Test (FRT). Three stability indexes - overall (OSI), anterior-posterior (APSI), and medial-lateral (MLSI) - both with eyes open (EO) and closed (EC) were analyzed. The impact of vision on balance was calculated as EC-EO. Also effect size was calculated and evaluated.

Results: All of the parameters differed significantly between groups in favour of YW. The largest difference in significance as well as effect size was noted for FRT, $\mathrm{p}<0.001$ and 1.86 respectively. Tries measure the impact of vision on balance (EC-EO). The results concerning tries with EC-EO showed the strongest discrimination between groups - OSI $p=0.0088$ (relative difference $0.23 \pm 0.26$ ) and APSI $p=0.0268$ (relative difference $0.17 \pm 0.2$ ). YW had a significantly better outcome.

Conclusions: YW had better results in all of the parameters taken into consideration, with most of them being significant. This confirms that all of the regressive changes appearing with age influence balance. Lack of visual input (EC) in OW caused significantly worse results in most of the measured parameters, showing that vision is a very important factor for balance maintenance in the elderly.
\end{abstract}

\section{Key words: Balance - Aging - Physical activity - Stabilometry}

\section{Introduction}

The human body is constantly trying to maintain equilibrium regarding balance in the upright position. Postural instability may be caused by inefficiency of each balance control component: vision, vestibular and proprioceptive information $[4,15]$. Unfortunately, postural control decreases with age $[4,26]$. The complex and diversified etiology of balance disorders causes many diagnostic and treatment difficulties. Improper work of control mechanisms responsible for maintaining balance in the older population results in functional limitations or disabilities, e.g. postural instability. The same symptoms may occur due to damaged receptors, improper nervous impulse conduction up to the central nervous system (CNS) as well as wrong impulse interpretation in the CNS or conduction down to the effectors. Additionally, in older persons, the reaction time is prolonged, which widens the margin of safety [5]. According to Nitz et al. [26], anterior-posterior postural instability starts at 40 years old in women and worsens with age.
Maintaining balance on an unstable surface has a tremendous impact on preventing risk of fall in daily living. Falls in the elderly are becoming a very alarming social issue in the developed western countries such as Australia and the USA due to their increasing frequency in successive years. In 2004, the number of deaths due to unintentional falls was 41,000 and ten years later in 2013 it was almost 57,000. The 2013 data show that each year 2.5 million older people are treated in emergency departments due to fall injuries. Approximately $20 \%$ of accidental falls require medical attention, and 5\% result in serious injuries, such as fractures $[8,17,25,31]$.

Stabilometry is a widely used method of examining humans' stability $[13,23]$. The platform on which the patients stand measures the centre of pressure (COP). Most of the stabilometry protocols use stable platforms which do not describe the complexity of the balance mechanism $[9,36]$. The most important part of the definition states that balance is resistance against disruptions coming from both inside and outside of the body (environment changes) as well as the interaction of these two factors $[4,7]$. 
Examination performed on an unstable (moveable) platform seems to be a very important link in supplementing the analysis of balance and an attempt to understand its characteristics better $[10,27,38]$.

Based on aforementioned premises concerning both high prevalence of falls in the elderly population together with all negative health and social complications resulting from them, it seems important to reveal factors differentiating young and older women's balance. Once identified they can form a basis for further cause and effect considerations helping to create protocols for preventive interventions, attempting to resolve the issues mentioned above.

The aim of this study was to assess and compare postural stability of women over 60 and young women on an unstable platform.

Based on current knowledge the authors hypothesized the following:

1. Involutional changes occurring in the human body with age have a negative influence on postural stability.

2. Vision is one of the sensory inputs that play a crucial role in balance maintenance, and can compensate for other inputs' dysfunction.

3. Postural stability can be described and assessed better when using dynamic measurements in contrast to static.

\section{Material and methods}

Seventy-three female participants joined the study after oral informed consent was obtained. They were divided into two groups: older $(\mathrm{OW})$ and young women $(\mathrm{YW})$. All members of the OW group were attending PA classes within the University of the Third Age on a daily basis. The other group consisted of students from the Department of Rehabilitation who had organized PA classes in the course of their studies. Participation groups differed significantly from each other in the following respects: age, body height, body mass and body mass index (BMI). Participants' demographics are depicted in Table 1.

All participants underwent a physical examination performed by a medical doctor specialized in geriatrics to exclude all possible contraindications influencing balance control. Exclusion criteria consisted of ECG disorders, cranial nerve dysfunction, dural signs, positive cerebellum tests, gait disorders (Romberg's sign, Unterberger's test, Babiński-Weil test, Fukuda test straight line walking test) and any medication that potentially could influ- ence balance control. Participants could not compete in professional sport.

Participant's history and physical examination were conducted by a medical doctor. Also morphological characteristics (body height, body weight and body mass index - BMI) and postural stability were measured.

The latter was obtained by using a Biodex Balance System SD (BBS) (Biodex, USA) both with a stable and an unstable platform. The protocol consisted of five tests, which is depicted below in Figure 1.

\section{PST}

PST emphasizes a patient's ability to maintain centre of balance. The patient's score on this test assesses deviations from centre; thus a lower score is more desirable than a higher score.

The PST was performed in three different ways: one with stable ground with eyes open (EO) and eyes closed (EC), second on unstable ground (level 4) with EO and biofeedback and the third attempt was performed on un unstable ground (level 8) with EC.

\section{FRT}

The FRT test allows identification of potential fall candidates. Test results are compared to age-dependent normative data (provided by the manufacturer; also the authors possessed their own normative data from previous research on a Polish population). Scores higher than normative values suggest further assessment for lower extremity strength, proprioception, and vestibular or visual deficiencies. In the FRT the platform's instability changed from level 6 to 2 (less to more unstable).

All BBS tests have been described in more detail elsewhere [38].

\section{PST}

3 attempts, 20s each, 10s break in between each attempt;

1st stable (rigid) ground EO and EC;

2nd unstable ground (level 4) EO with biofeedback;

3 unstable ground (level 8) EC;

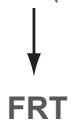

unstable ground, from level 6 to 2 ;

Fig. 1. Examination protocol chart (PST - Postural Stability Test; FRT - Fall Risk Test; EO - eyes open, EC - eyes closed)

Table 1. Participants' demographics (mean \pm SD) OW - women over 60 and YW - younger women

\begin{tabular}{lccccc}
\hline Group & $\mathrm{n}$ & Age [years] & Body height $[\mathrm{cm}]$ & Body mass $[\mathrm{kg}]$ & BMI \\
\hline OW & 30 & $68.3 \pm 7.43$ & $160.6 \pm 6.95$ & $69.2 \pm 12.23$ & $26.8 \pm 4.60$ \\
YW & 43 & $20.2 \pm 1.75$ & $168.1 \pm 6.08$ & $59.6 \pm 5.44$ & $21.1 \pm 1.77$ \\
\hline
\end{tabular}


Figure 1 presents the examination protocol. The authors proposed a ratio of differences between the $\mathrm{EC}$ and EO. Biodex Balance System SD (BBS) is made of a platform, which can work in two modes - stable and unstable (12 levels of movement). Medical System Biodex, Inc. (v. 1.3.4) software allows one to measure the angulation in each of the main axes $(\mathrm{x}, \mathrm{y}, \mathrm{z})$, also creating three stability indexes (SI): overall (OSI), anterior-posterior (APSI) and medial-lateral (MLSI). Higher values represent more sway, which indicates balance control impairments.

The protocol consisted of five tests: three 20-second attempts with a 10-second break in between, the Postural Stability Test (PST) and the Fall Risk Test (FRT). The PST was performed in three different ways: one with stable ground with eyes open (EO) and eyes closed (EC), second on unstable ground (level 4) with EO and biofeedback and the third attempt was performed on un unstable ground (level 8) with EC. In the FRT the platform's instability changed from level 6 to 2 (less to more unstable).

All data were analyzed with Statistica (v. 10) software. The parameters were analyzed using the Shapiro-Wilk test and ANOVA. Tukey's (honestly significant difference, HSD) post hoc test was performed with the significance level at $\alpha=0.05$. Effect size was estimated using Cohen's d, where higher values indicate a stronger effect between variables.

The study was approved by the Ethics Committee at Józef Piłsudski University of Physical Education in Warsaw.

\section{Results}

The results represent values for SI from the PST and FRT performed with EO and EC. All of the parameters differed significantly. The YW group had better results than OW in all of the measurements for mean values and standard deviation (Table 2).
The largest difference in significance was noted for the FRT $(\mathrm{p}<0.001)$, where the platform was free to move gradually from level 6 to 2 (more to less stable).

The effect size, which is the quantitative measure of the strength of each parameter, was the highest for FRT again (1.86). It is also confirmed by low values of the coefficient of variation, which is a measure of results' dispersion. Higher dispersion of values can be observed in all tries in OW. EO tries brought even higher results, whereas $\mathrm{CV}$ was quite similar between groups and values were moderate when EC testing was involved. The smallest differences were noted for MLSI EC (0.58) - the parameter was still significantly different between groups.

Both groups had the same increase in values (4-5 times) when comparing indexes from tests with EO and EC. The numbers themselves were greatly different, but the ratio remained quite similar.

When comparing the ratio of differences between the EC and EO with stability indexes (OSI, APSI and MLSI) between the groups, significance was found only in two OSI OW $1.99 \pm 1.26$, YW $1.37 \pm 0.70(\mathrm{p}=0.0088$, relative difference $0.23 \pm 0.26$ ) and APSI OW $0.97 \pm 0.67$, YW $1.48 \pm 1.24(\mathrm{p}=0.0268$, relative difference $0.17 \pm 0.2)$. The last parameter MLSI (OW $1.09 \pm 0.84$ and YW $0.77 \pm 0.58)$ was close to significance $(p=0.056)$. This proves a strong discrepancy between young and older women when vision is the considered variable.

Larger differences can be seen in all of the attempts with eyes closed. This fact is emphasized by the results of the effect size. Here, special attention should be paid to the difference between eyes closed and eyes open (a value obtained by subtracting results from EC and EO attempts) where OSI and APSI changed significantly between groups (Figure 2). This parameter may be very useful, especially when the elderly group's results are concerned, since it estimates the influence of vision.

Table 2. Participants' baseline measures for OW (older women) and YW (younger women) (mean \pm SD). Tukey's test (HSD)

\begin{tabular}{lccccccc}
\hline Stability Index & OW $(\mathrm{n}=30)$ & $\mathrm{CV} \%$ & $\mathrm{YW}(\mathrm{n}=43)$ & $\mathrm{CV} \%$ & $\begin{array}{c}\text { Effect size } \\
\text { Cohen’s d }\end{array}$ & $\begin{array}{c}\text { OW-YW } \\
\text { relative differences [\%] }\end{array}$ & HSD \\
\hline OSI EO & $0.57 \pm 0.42$ & 73.7 & $0.34 \pm 0.16$ & 47 & 0.79 & $0.23 \pm 0.26$ & 0.004 \\
APSI EO & $0.44 \pm 0.35$ & 79.5 & $0.27 \pm 0.15$ & 55.5 & 0.68 & $0.17 \pm 0.2$ & 0.008 \\
MLSI EO & $0.25 \pm 0.20$ & 80 & $0.16 \pm 0.09$ & 56.2 & 0.62 & $0.09 \pm 0.55$ & 0.016 \\
OSI EC & $2.56 \pm 1.15$ & 44.9 & $1.72 \pm 0.69$ & 40.1 & 0.91 & $0.84 \pm 0.46$ & 0.0007 \\
APSI EC & $1.92 \pm 1.14$ & 59.4 & $1.23 \pm 0.69$ & 56.1 & 0.75 & $0.69 \pm 0.45$ & 0.004 \\
MLSI EC & $1.34 \pm 0.85$ & 63.4 & $0.93 \pm 0.56$ & 60.2 & 0.58 & $0.41 \pm 0.29$ & 0.023 \\
FRT EO & $2.18 \pm 0.85$ & 39 & $1.08 \pm 0.33$ & 30.5 & 1.86 & $1.1 \pm 0.52$ & 0.0002 \\
\hline
\end{tabular}

All values mean $\pm \mathrm{SD}$, OSI - overall stability index, APSI - anterior-posterior stability index, MLSI - medial-lateral stability index, EO - eyes open, EC - eyes closed, FRT - fall risk test. Effect Size (Cohen's d), Tukey's test (HSD) statistical significance p $<0.05$, CV - coefficient of variation. 


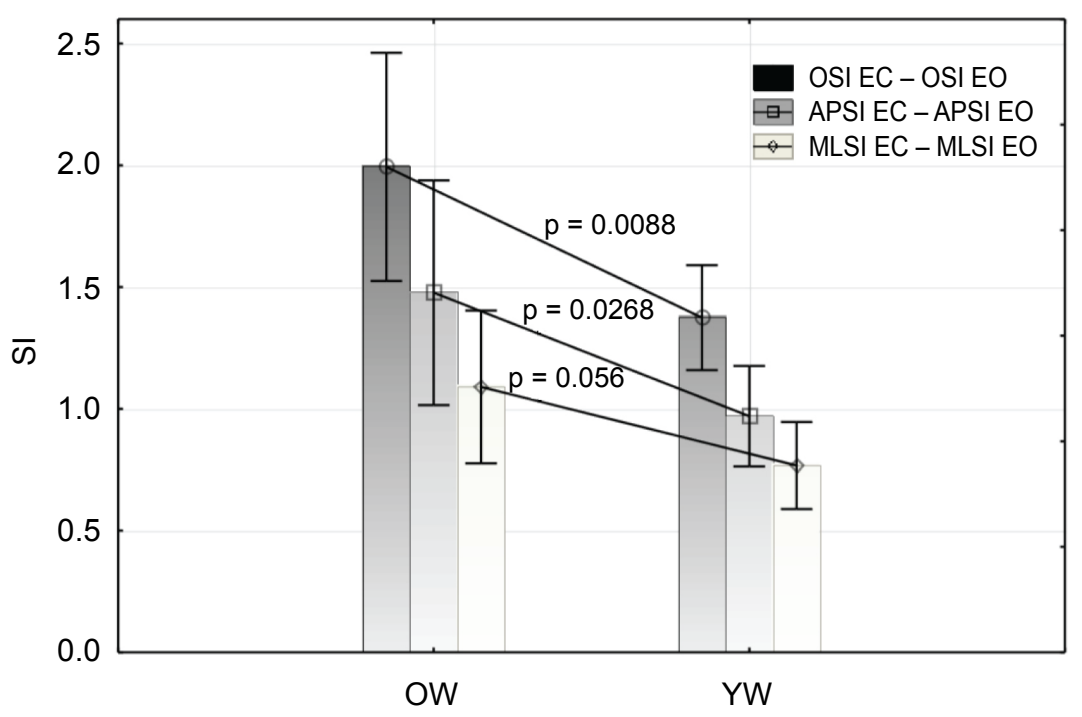

Fig. 2. Differences between particular stability indexes with eyes closed and eyes open

\section{Discussion}

Postural stability decreases with age - this has been conclusively demonstrated by many researchers $[8,15$, $24,34]$. The authors hypothesized that this process is due to involutional changes occurring in the human body with age. The main factors responsible for this are as follows: decreases in muscle mass and strength, which are caused by changes in the nervous system and muscles themselves due to decreased participation in physical activity $[6,18$, 30]. Such factors as vision, vestibular, loss of peripheral sensation, and decrease in reaction time were noted too $[21,37]$.

The results showed significant differences in all of the postural stability parameters between young and elderly women. Considering all the age-related factors that influence balance, the results support findings presented by others [34].

Postural stability has become a very popular subject in recent years. The main reason is the possibility to predict balance impairments which can lead to falls, especially in the elderly $[16,24,34]$. Due to a consistent increase of falls, researchers started to identify factors that influence balance as well as seeking interventions improving postural stability [10]. All such preventive actions could decrease the cost of medical care $[11,35]$.

Weirich et al. divided women in their research into three age categories (young, middle-aged and late middleaged) and proved that postural stability worsens with age - especially when the examination consisted of dynamic tries. They identified several predictors of falls and noted that correlations between them and balance control were easier to anticipate as age increased [36].
Although the methodology of that study was different, the same phenomenon was observed in this study, where YW results were better compared with OW in all examined tries, namely the OSI, APSI, and MLSI with both EO and EC as well as the FRT.

Darwesh et al. compared stability in two groups (young and older) using the BBS. They concluded that regarding the stability indexes the results were significantly higher in the older group. Since the authors used the same technology for measurements (BBS) as ours, the results could be compared directly. That is for OSI YW $0.75 \pm 0.35$ and OW $1.17 \pm 0.35(\mathrm{p}<0.002)$, APSI YW $0.81 \pm 0.3$ and OW $1.48 \pm 0.35(\mathrm{p}<0.001)$ and finally MLSI YW $1.13 \pm 0.47$ and OW $1.7 \pm 0.48(\mathrm{p}<0.0009)$ with the last test not being significant. The absolute values reported by Darwesh et al. are higher for both groups than in the current study, although the protocols were similar. The only difference noted was the resting time - one minute sitting in the study by Darwesh et al. versus 15 seconds standing on the platform in the current study. The rest of the parameters were the same, i.e. stability level of the platform set at 8 (most stable), eyes open, 3 attempts, 20 seconds each [10]. Their explanation for this phenomenon was that an increased effort was required to keep balance in the older group. Other authors who came to similar conclusions provided different reasoning: decreased scores of the Berg scale, increased lower extremity muscle contraction while standing, profound ankle muscular co-activation, specific motor and sensory deficits such as weakness of lower limb muscles, peripheral hypoesthesia, visual impairment and decreased reaction time [3, 14, 19, 21].

All cited studies positively confirmed the hypothesis, by showing better results in the younger groups. The main causes led towards structural and functional changes 
within the muscle itself, and also in the nervous system controlling it, i.e. slower reaction time, etc.

The second hypothesis stated that visual input is most important for balance maintenance together with its compensatory capabilities compared to other sensory input systems.

As mentioned before, balance and posture are regulated by various multi-sensory processes. According to Peterka et al., the contribution of the main systems is as follows - somatosensory (70\%), vestibular (20\%), vision $(10 \%)$. This applies to healthy people in a well-lit environment, standing on a solid surface. When the conditions change to an unstable surface, vestibular and visual system inputs increase at the cost of somatosensory input [27]. This statement is reflected in the current results. Basically both groups showed 4-5 times higher values in EC tries, compared to EO, for instance OSI for OW with EO $0.57 \pm 0.42$ vs. EC $2.56 \pm 1.15$ or YW with EO $0.34 \pm 0.16$ vs. EC $1.72 \pm 0.69$.

Others state that about $80 \%$ of our sensory perception information is collected by the visual system. It is used for recognising, analysing and choosing the correct strategy for maintaining balance in the upright position. If one of the subsystems reduces its influence on balance, others compensate for that loss. In the case of visual disturbance the vestibular system is the first one to do so $[12,29]$.

The literature shows disagreement regarding the role of vision. Researchers tried to resolve this matter based on studies with blinded subjects as participants. Some studies show that blind subjects can maintain equilibrium better than sighted subjects [28].

Schmidt et al. compared the results of congenitally blinded and sighted subjects. Their study led to the conclusion that blind subjects, regardless of EO or EC, behaved the same way as sighted subjects with EC and the strategies for maintaining balance were similar [29].

The preceding conclusions emphasized the role of vision in balance maintenance. As people age, the loss of vision is significant, resulting in an increase of falls in many cases $[20,34]$. This may be a crucial factor for explaining the balance test results getting worse in the elderly in general, as well as in the current study.

Despite the contradictory results cited at the beginning of this section, most researchers emphasized the importance of vision in equilibrium, especially when other sources of necessary information are compromised. These statements are in agreement with the authors' hypothesis.

Maintaining balance in dynamic conditions requires more balance control involvement (vision, vestibular and somatosensory); thus, it is more relevant in exposing postural stability impairments or deficiencies and their relation to risk of falls [27, 38]. Turbański et al. performed a six-week somatosensory-exercise programme using unstable shoe constructions, and compared the results from both static and dynamic platforms. They found that with dynamic training, in one of the experimental groups (out of two) dynamic measurements were significant, while the results from a static platform remained unchanged in both groups [12].

Stemplewski et al. conducted a study where participants were asked to stand still and relaxed under two conditions - a stable and unstable base of support [31]. Nominal differences between mean values of particular parameters were as follows: general stabilometry parameters $114.9 \%$, ML direction parameters $62.8 \%$ and AP direction parameters $93.9 \%$, which gives $90.5 \%$ as the average value between unstable and stable ground. Comparison of parameters in varied conditions showed higher values of general stabilometry parameters $(\mathrm{p}<0.001)$ as well as ML and AP (at least $\mathrm{p}<0.01$ ) in unstable conditions (excluding one of the tested parameter in ML). The authors state that the risk of falls increases almost two-fold under unstable conditions. The examined population was in good general health, so the values might be even higher if the study were cross-sectional.

The above-mentioned results support the hypothesized superiority and importance of dynamic measurements over stable conditions. The authors emphasize its ability to act as an early predictor of falls due to its accuracy in the medio-lateral plane, which will be discussed later in more detail.

When it comes to comparing SI, their role and importance in measuring subject's stability, the literature includes very diverse statements. The authors dispute which of the three (OSI, APSI, MLSI) are the most suitable for stability analysis.

Aydog et al. examined the reliability of the BBS indices in visually impaired athletes. The APSI and OSI were more reliable than others. The MLSI was not highly reliable, but only fairly reliable. The main conclusion emphasized the OSI as the best indicator for overall stability [1]. Contrary to the outcomes mentioned above, Baloh et al. stated that ML results are better predictors of balance impairments than AP indices [2]. Also the oldest group participating in the research revealed the strongest correlations between stability parameters.

MLSI is considered the most important parameter in balance maintenance. There is an evident tendency for balance control in this plane to become more problematic with increasing age [33]. This phenomenon, with borderline significance $(p=0.056)$, can also be observed in the current study, where differences of MLSI for OW and YW were greater $(0.25 \pm 0.2$ vs. $0.16 \pm 0.09$ for $\mathrm{EO}$ and $1.34 \pm 0.85$ vs. $0.93 \pm 0.56$ for $\mathrm{EC}$ respectively for $\mathrm{OW}$ vs. $\mathrm{YW}$ ), regardless of EC/EO. Lack of visual input emphasized it even more in $\mathrm{OW}$, as discussed earlier.

Based on results from the current study and data provided by others, one can conclude that postural balance control 
is a complex and a complicated mechanism controlled by different variables [7]. In summary, dynamic examination protocols seem to be more adequate for daily living activities which are based on constant adjustment to a changeable environment. Perhaps dynamic tries can reveal balance deficiencies sooner than static testing. The most demanding tries among dynamic protocols are the ones where the level of instability changes within the same try. The Fall Risk Test (FRT) differentiates participants the most.

As the Fall Risk Test puts more demand on the vestibular system, it may be considered as more sensitive than other tests in predicting balance disorders. Potentially FRT can be used as a screening tool for early stages of balance disorders.

The authors found a number of limitations of their study. Both groups were physically active on a daily basis. OW members were students of the University of the Third Age, so it can be assumed that they are more active, both physically and socially, than the average peer from assisted living facilities for instance. This also applies to the YW, who were students at the University of Physical Education, where they take part in many PA classes during the course of studies. Taking these two facts into consideration, the results from this study should not be generalized to the whole population.

Future studies should compare stability indexes of active and inactive (sedentary) older persons. Possibly this would reveal the influence of PA on balance, which is crucial for wellbeing.

\section{Conclusions}

1. YW had better results in all of the parameters taken into consideration, with most of them being significant. This confirms that all of the regressive changes appearing with age influence balance.

2. Lack of visual input (EC) in OW caused significantly worse results in most of the measured parameters, showing that vision is a very important factor for balance maintenance in the elderly.

3. Effect size is a good supplementary factor to other statistical tools. It emphasizes the strength of the phenomenon taking place.

\section{References}

1. Aydog S.T., Aydog E., Çakci A., Doral M.N. (2004) Reproducibility of postural stability scores in blind athletes. Isokinet. Exerc. Sci., 12: 229-232.

2. Baloh R.W. (1998) Differentiating between peripheral and central causes of vertigo. Otolaryngol. Head Neck. Surg., 119(1): 55-59.
3. Benjuya N., Melzer I., Kaplanski J. (2004) Aging induced shifts from a reliance on sensory input to muscle cocontraction during balanced standing. J. Gerontol. A Biol. Sci. Med. Sci., 59A(2): 166-171.

4. Błaszczyk J.W., Cieślinska-Świder J., Plewa M., Zahorska-Markiewicz B., Markiewicz A. (2009) Effects of excessive body weight on postural control. J. Biomech., 42(9): 1295-1300.

5. Błaszczyk J.W., Lowe D.L., Hansens P.D. (1993) Postural sway and perception of the upright stance stability borders. Perception, 22: 1333-1341.

6. Błaszczyk J.W., Michalski A. (2006) Ageing and postural stability. SPCT13: 11-14.

7. Błaszczyk J.W. (2008) Sway ratio - a new measure for quantifying postural stability. Acta Neuroobiol. Exp. (Wars.), 68: 51-57.

8. Choy N.L., Brauer S., Nitz J. (2003) Changes in Postural Stability in Women Aged 20 to 80 Years. J. Gerontol. A Biol. Sci. Med. Sci., (58): 525-530.

9. Daniel F., Vale R., Giani T., Bacellar S., Dantas E. (2010) Effects of a Physical Activity Program on Static Balance and Functional Autonomy in Elderly Women. Maced. J. Med. Sci., 3(1): 21-26.

10. Darwesh A., Elsayed E., Abbas H., et al. Balance Deficits in Elderly Population. Middle East J Sci Res 2014:21(1):1-6.

11. Davlin-Pater C. (2010) The Effects of Visual Information and Perceptual Style on Static and Dynamic Balance. Motor Control, 14(3): 632-670.

12. Friedrich M., Grein H.J., Wicher C., Schuetze J., Mueller A., Lauenroth A., Hottenrott K., Schwesig R. (2008) Influence of pathologic and simulated visual dysfunctions on the postural system. Exp. Brain Res., 186(2): 305-314.

13. Held-Ziółkowska M. (2006) Postural Stability. Mag. Otolaryngol., 52(18): 39-46.

14. Hojjati Z., Sheikhpour L. (2013) Seated Exercise Therapy Improves Posture and Balance in Hyperkyphotic Elderly Females, a Randomized Control Trial. World Appl. Sci. J., 24(3): 331-335.

15. Horak F.B. (2006) Postural orientation and equilibrium: what do we need to know about neural control of balance to prevent falls? Age Ageing 35(2).

16. Kaeding T.S. (2009) Sarcopenia and whole body vibration training. Z Gerontol. Geriatr., 42(2): 88-92.

17. Kannus P., Sievänen H., Palvanen M., Järvinen T., Parkkari J. (2005) Prevention of falls and consequent injuries in elderly people. Lancet, 366(9500): 1885-1893.

18. Kim B., Robinson C. (2005) Postural control and detection of slip/fall initiation in the elderly population. Ergonomics, 48(9): 1065-1085.

19. Laughton A., Slavin M., Katdare K., Nolan L., Bean J.F., Kerrigan D.C., Phillips E., Lipsitz L.A., Collins J.J. (2003) Aging, muscle activity and balance control: 
Physiologic changes associated with balance impairment. Gait Posture, 18(2): 101-108.

20. Lee H.K.M., Scudds R.J. (2003) Comparison of balance in older people with and without visual impairments. Surg. Endosc., 32: 643-649.

21. Lord R., Ward A. (1994) Age-associated differences in sensori-motor function and balance in community dwelling women. Age Ageing, 23(6): 452-460.

22. Melzer I., Benjuya N., Kapłański J. (2005) Effect of physical training on postural control of elderly. Harefuah, 144(12): 839-844.

23. Mraz M., Curzytek M., Skrzek A., Zubińska B. (2008) Postural stability in patients with nervous system impairment. Acta Bio-Opt. Inform. Med., 14(2): 111-114.

24. Nagy E., Feher-Kiss A., Barnai M., Domján-Preszner A., Angyan L., Horvath G. (2007) Postural control in elderly subjects participating in balance training. Eur. J. Appl. Physiol., 100(1): 97-104.

25. National Center for Injury Prevention and Control. Webbased Injury Statistics Query and Reporting System (WISQARS) Centers for Disease Control and Prevention, Atlanta, GA, USA 2015. Available at http://www. cdc.gov/injury/wisqars/. Accessed January 21, 2016.

26. Nitz J., Choy N.L., Isles R. (2003) Medial-lateral postural stability in community-dwelling women over 40 years of age. Clin. Rehabil., 17(7): 765.

27. Peterka R.J. (2002) Sensimotor Integration in human Postural Control. J. Neurophysiol., 88: 1097-1118.

28. Pyykkö I., Vesikivi M., Ishizaki H., Magnusson M., Juhola M. (1991) Postural control in blinds and in Usher's syndrome. Acta Otolaryngol. Suppl., 481: 603-606.

29. Schmid M., Nardone A., De Nunzio A.M., Schmid M., Schieppati M. (2007) Equilibrium during static and dynamic tasks in blind subjects: no evidence of cross-modal plasticity. Brain, 130(8): 2097-2107.

30. Sherrington C., Lord S.R., Finch C. (2004) Physical activity interventions to prevent falls among older people: update of the evidence. J. Sci. Med. Sport, 7(1): 43-51.
31. Stemplewski R., Salamon A., Maciaszek A. (2013) Static body balance posturographic values in medio-lateral and anterior-posterior directions in conditions of unstable base of support in men older than 70 years. SPCT, 13: 85-87.

32. Stevens J.A., Ballesteros M.F., Mack K.A., Rudd R.A., DeCaro E., Adler G. (2012) Gender differences in seeking care for falls in the aged Medicare population. Am. J. Prev. Med., 43(1): 59-62.

33. Stunkiers D.L., St George R., Lord S.R. (2015) Balance disorders in the elderly. Clin. Neurophysiol., 38: 467.

34. Thomas D. (2007) Loss of skeletal muscle mass in aging: examining the relationship of starvation, sarcopenia and cachexia. Clin. Nutr. (Edinburgh, Scotland), 26(4): 389-399.

35. Turbański S., Schmidtbleicher D. (2010) Postural control depends on testing situation. Sportverletz Sportschaden, 24(3): 123-128.

36. Weirich G., Bemben D., Bemben M. (2010) Predictors of balance in young, middle-aged, and late middle-aged women. J. Geriatr. Phys. Ther., 33(3): 110-117.

37. Wiszomirska I., Kaczmarczyk K., Błażkiewicz M., Wit A. (2015) The impact of a vestibular-stimulating exercise regime on postural stability in people with visual impairment. BioMed. Res. Int., 1-8.

38. Wiszomirska I., Kaczmarczyk K., Zdrodowska A., Błażkiewicz M., Ilnicka L., Marciniak T. (2013) Evaluation of static and dynamic postural stability in young, elderly and with vision loss women. Adv. Rehabil., 27(3): 33-39.

\section{Received 29.01.2018 \\ Accepted 16.09.2018}

(C) University of Physical Education, Warsaw, Poland 